\title{
Prevalencia de resistencia primaria en pacientes con infección reciente por VIH-1 en Chile
}

\author{
ALEJANDRO AFANI S. ${ }^{1}$, CARLOS BELTRÁN B. ${ }^{2}$, \\ ANA MARÍA GALLARDO O. ${ }^{1,3}$, PATRICIA ROESSLER V. ${ }^{1}$, \\ WILLIAM ACEVEDO M. ${ }^{\text {la }}$, PATRICIA VÁSQUEZ T. ${ }^{4}$
}

\section{Prevalence of primary antiretroviral resistance among HIV infected patients in Chile}

Background: The main cause of virological failure during AIDS treatment is the resistance to antiretroviral medications (ARV). Aim: To search for mutations associated with $A R V$ resistance in recently HIV-1 infected patients naïve to treatment, in Chile. Material and Methods: Patients over 18 years old with HIV-1 infection, naïve to anti-retroviral drugs before the study were included. Patients with CD4 cell counts less than $200 \mathrm{cells} / \mathrm{mm}^{3}$, viral load below 2.000 copies/mL or any condition indicative of advanced AIDS were excluded. Criteria for diagnosis of recent infection $(<18$ months) were a previous negative test for HIV antibodies or a history of an acute retroviral syndrome in the past 18 months. Resistance to drugs was analyzed using the TRUGENE ${ }^{\text {tm }} H I V-1$ assay from Bayer and the OpenGene DNA sequencing system. Results: Ninety nine percent of patients had at least one mutation, $27 \%$ had 4 or more mutations, but high level resistance to $A R V$ was found only in $2.7 \%$ of cases. Point mutations for non nucleoside reverse transcriptase inhibitors (NNRTI) were detected in $4.1 \%$ of cases (K103N in 1 patient, V179D in 2 patients), for nucleoside reverse transcriptase inhibitors (NRTI) in $8.1 \%$ of cases (T215S in 1 patient, V118I in 4 patients, M41L in 1 patient) and for protease inhibitors (PI) in $1.3 \%$ of cases. All mutations detected in the protease gene were secondary. Of these, the most common were L63P/T (38 patients), L10I/V (27 patients) and V77I (26 patients). Resistance to two or more antiretroviral classes was not detected. Conclusions: This study supports that, by now, primary resistance has a low prevalence in Chile. Therefore, a genotyping test before starting antiretroviral therapy is not necessary.

(Rev Med Chile 2010; 138: 669-676).

Key words: Antiretroviral therapy; Drug resistance, Genotype.



S egún el programa de SIDA de las Naciones Unidas, el número estimado de personas con infección por el virus de la inmunodeficiencia humana $(V I H)$ en todo el mundo en el año 2007 fue de 33,2 millones ${ }^{1}$.

En Chile, la epidemia de VIH/SIDA se inició en 1984 . Entre 1990 y 2006 han muerto 5.710 personas por SIDA y, hasta el 2007, se han notificado 18.552 casos de VIH/SIDA ${ }^{1}$.
La terapia antirretroviral de alta actividad (TARV) ha logrado disminuir drásticamente la morbimortalidad de la infección por VIH. En Chile, la TARV comienza a administrarse en el sistema público en 1993 en forma de monoterapia con zidovudina. En 1997 se introduce la biterapia, que se mantiene hasta 1999, cuando se inicia la entrega de triterapia en forma parcial, y en 2002 con una cobertura de $100 \%$. Se utilizan principal- 
mente combinaciones de drogas antirretrovirales (ARV) pertenecientes a las siguientes familias: i) inhibidores de la transcriptasa reversa análogos de nucleósidos (NRTI), ii) inhibidores de la transcriptasa reversa no análogos de nucleósidos (NNTRI), iii) inhibidores de proteasa (IP) ${ }^{2,3}$.

Uno de los principales problemas asociados a la TARV es la falla virológica, es decir, carga viral sobre 1.000 copias/ml después de 24 semanas de inicio de la terapia o después de haber sido indetectable 4 . La principal causa de falla virológica es la aparición de resistencia a las drogas ARV, ocasionada por la aparición de mutaciones a nivel de los genes de la transcriptasa reversa y de la proteasa viral. Este problema se origina por la asociación de las características propias del virus, junto a una inadecuada supresión viral y uso de monobiterapia previos. La resistencia viral que aparece bajo la presión de las ARV se denomina resistencia secundaria. La resistencia primaria ocurre en pacientes vírgenes a tratamiento, como resultado de la transmisión de virus resistente ${ }^{5,6}$.

La prevalencia de resistencia primaria a nivel mundial es variable, y va a depender de factores como el nivel de exposición de la población a los distintos ARV, vías de transmisión, tiempo de infección previo al desarrollo de genotipificación, definiciones de resistencia en los distintos estudios, etc ${ }^{7}$. Los estudios para evaluar la prevalencia de resistencia primaria en una determinada población de infectados deben realizarse idealmente en individuos en los cuales se tenga certeza de infección reciente ( $<18$ meses), y que no hayan recibido TARV.

Los índices de resistencia primaria son altos en Estados Unidos y Europa, probablemente debido a que han tenido acceso por más tiempo a las ARV. Durante el período 2003-2004, los estudios realizados en Europa y Norteamérica reportan índices de resistencia primaria entre 3\% y $29 \%{ }^{7-}$ ${ }^{12}$. En estas regiones, se recomienda realizar un test de resistencia a los pacientes naive a TARV. Se ha sugerido su aplicación con prevalencia de resistencia primaria igual o mayor al $5 \%$ en la población de referencia. La mayoría de los países de Europa y Norteamérica, recomiendan realizar tests de resistencia previo al inicio de TARV, ya que superan el límite de 5\% de prevalencia de resistencia primaria ${ }^{7-9}$.

En Latinoamérica la información es limitada, y muy variable, dependiendo de la región. En Chile, existen pocos datos a cerca de la prevalencia de resistencia primaria. El objetivo de este trabajo es establecer la prevalencia de resistencia primaria en una población chilena de pacientes con infección reciente por VIH naive a TARV, en 30 meses de estudio.

\section{Material y Método}

Se realizó un estudio descriptivo prospectivo a través de un proyecto colaborativo del Ministerio de Salud, y la Sección Inmunología del Departamento de Medicina del Hospital Clínico de la Universidad de Chile, donde se reclutaron pacientes desde julio de 2006 hasta diciembre de 2008 (30 meses).

Se incluyeron pacientes mayores de 18 años, con infección reciente por VIH-1 confirmada en el Centro Nacional de Referencia para el VIH del Instituto de Salud Pública, que no hubieran recibido ARV. Se excluyeron aquellos pacientes con recuentos de linfocitos $\mathrm{CD} 4^{+}$inferiores a 200 células/mL, carga viral inferior a 2.000 copias/ $\mathrm{mL}$, o con alguna condición indicadora de SIDA. La definición de infección reciente se basó en: serología para VIH negativa previa y/o cuadro clínico de un síndrome retroviral agudo en los 18 meses previos.

A todos los pacientes se les entregó un consentimiento informado por escrito y una encuesta acerca del síndrome retroviral agudo, serología para VIH negativa previa, triterapia, donante o receptor de hemoderivados y hábitos sexuales.

El test de resistencia se realizó mediante el Ensayo TRUGENE ${ }^{\mathrm{tm}}$ HIV-1 (Bayer S.A.), y el sistema de secuenciamiento OpenGene DNA del Laboratorio de Medicina Molecular del Hospital Clínico de la Universidad de Chile. Mediante este sistema es posible detectar mutaciones en el genoma del VIH que confieren resistencia a ARV específicos. Se estudiaron las mutaciones en el gen pol del virus, específicamente las regiones de la transcriptasa reversa y proteasa viral. Los patrones de resistencia son definidos según la base de datos de la Universidad de Stanford ${ }^{13,14}$.

\section{Resultados}

\section{Características de los pacientes}

El test de genotipificación se realizó exitosamente en 74 pacientes chilenos con infección por 
VIH reciente. Las características de los pacientes se resumen en la Tabla 1. La mayoría de los pacientes fueron de sexo masculino $(87,8 \%)$, con una edad promedio de 32 años (rango de 18-58). Todos los pacientes estaban en etapa A de la clasificación del $\mathrm{CDC}^{4}$, el $45,5 \%$ en etapa $\mathrm{A} 1$, y el $54,5 \%$ en etapa $\mathrm{A} 2$, con un promedio de CD4 de 510 cél/ $\mathrm{ml}$ (rango 211-1450). El 63,5\% de los pacientes, presentó características clínicas de síndrome retroviral agudo, y $36,5 \%$ de ellos fueron asintomáticos con el antecedente de un ELISA para VIH (-) previamente.

\section{Análisis genotípico}

En relación al número de mutaciones encontradas en cada paciente, 73 pacientes $(98,7 \%)$ presentan al menos una mutación en el análisis genotípico, y 20 (27\%) de ellos presentaron 4 o más mutaciones (Figura 1).

De los 74 análisis genotípicos, $2,7 \%$ de los pacientes presentó mutaciones asociadas a resistencia elevada a las ARV. Los grados de resistencia (elevada, intermedia y baja) se refieren a la capacidad de reducir la sensiblilidad del virus a la droga.

\section{Tabla 1. Características generales de los pacientes}

\begin{tabular}{|c|c|}
\hline Características & Pacientes $(n=74)$ \\
\hline $\begin{array}{l}\text { Edad (años) } \\
\text { Promedio } \\
\text { Rango }\end{array}$ & $\begin{array}{l}32 \\
(18-58)\end{array}$ \\
\hline $\begin{array}{c}\text { Género }\left(n^{\circ}[\%]\right) \\
\text { Masculino } \\
\text { Femenino }\end{array}$ & $\begin{array}{l}66(87,8 \%) \\
8(12,2 \%)\end{array}$ \\
\hline $\begin{array}{l}\left.\text { Conducta de riesgo declarada ( } \mathrm{n}^{\circ}[\%]\right) \\
\text { Sexual (homobisexual) } \\
\text { Sexual (heterosexual) }\end{array}$ & $\begin{array}{l}57(77 \%) \\
17(23 \%)\end{array}$ \\
\hline $\begin{array}{l}\text { Criterios de selección }\left(\mathrm{n}^{\circ}[\%]\right) \\
\text { Sd. retroviral agudo } \\
\text { Asintomático con ELISA previo (-) }\end{array}$ & $\begin{array}{l}47(63,5 \%) \\
27(36,5 \%)\end{array}$ \\
\hline $\begin{array}{l}\text { Clasificación CDC } \\
\text { A1 } \\
\text { A2 }\end{array}$ & $\begin{array}{l}45,5 \% \\
54,5 \%\end{array}$ \\
\hline $\begin{array}{l}\text { Recuento CD4 } \\
\text { Promedio } \\
\text { Rango }\end{array}$ & $\begin{array}{l}510 \mathrm{cel} / \mathrm{ml} \\
(211-1450)\end{array}$ \\
\hline $\begin{array}{l}\text { Carga viral } \\
\text { Promedio } \\
\text { Rango }\end{array}$ & $\begin{array}{l}101.293 \text { copias } / \mathrm{ml} \\
(2.500-718.000 \text { copias } / \mathrm{ml})\end{array}$ \\
\hline
\end{tabular}

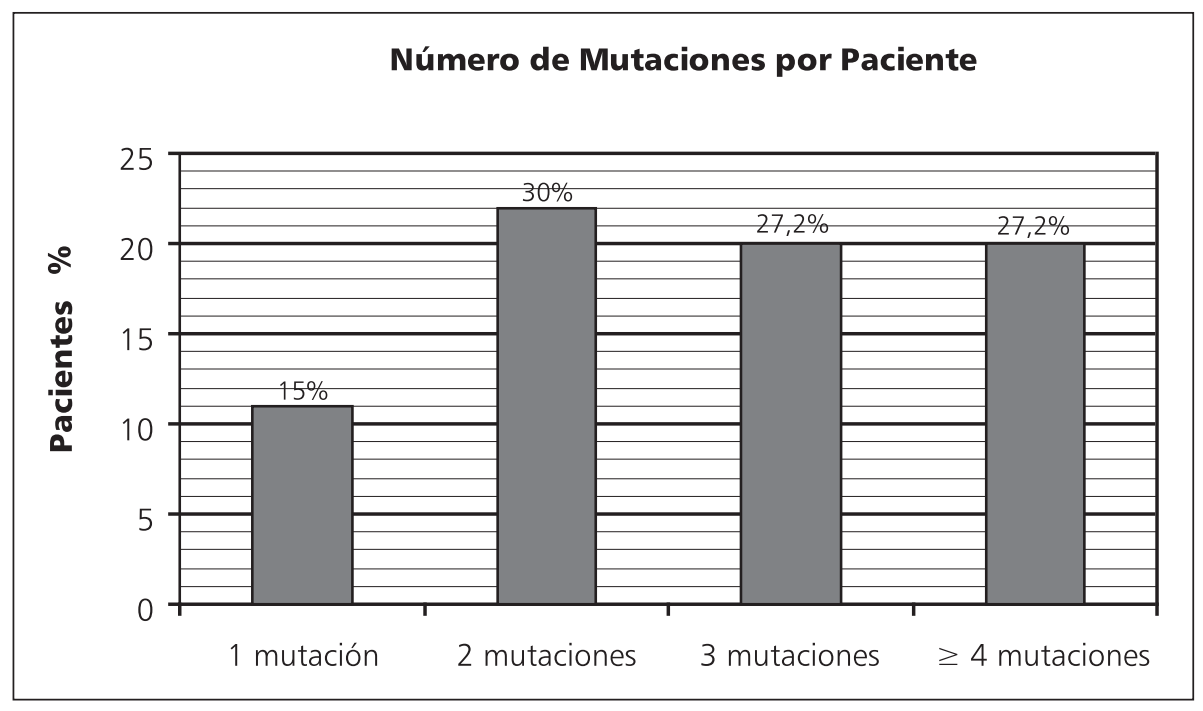

Figura 1. Número de mutaciones encontradas por paciente. 



Figura 2. Mutaciones encontradas agrupadas por familias de antirretrovirales.
Figura 3. Prevalencia de resistencia a drogas individuales.
Un total de 6 mutaciones fueron identificadas en la transcriptasa reversa. Dos de las mutaciones encontradas se asocian con resistencia a los NNRTI ( $4,1 \%$ de los casos); K103N en 1 paciente, que se asocia con alta resistencia, y V179D en 2 pacientes asociada a resistencia intermedia a este grupo de antirretrovirales. Las otras 4 mutaciones encontradas en el gen de la TR se asocian a resistencia intermedia-baja a los NRTI ( $8,1 \%$ de los casos): M41L (1 paciente), V118I (4 pacientes) y T215S (1 paciente). Sólo un paciente $(1,3 \%)$ presentó mutaciones asociadas a resistencia elevada a una droga de la familia de los IP (amprenavir/fosamprenavir), debido a la presencia simultánea de las mutaciones L10I, L19I, I62V y L63P.
En 13 pacientes se evidenció resistencia intermedia al grupo de los IP, debido a acumulación de mutaciones secundarias; 7 pacientes $(9,5 \%)$ a Saquinavir (L10I, I15V, K20R/M, I62V, A71T y V77I), 5 pacientes $(6,8 \%)$ a Tipranavir (L10V, I13V K20R, M36I, K43T), 3 pacientes $(4,1 \%)$ a Indinavir (L10I, K20R, A71T y V77I) y 3 pacientes $(4,1 \%)$ a Atazanavir (L10I, G16E, K20R, A71T, M36I) (Figuras 2 y 3 ).

Todas las mutaciones encontradas en el gen de la proteasa corresponden a mutaciones principalmente polimórficas, las que no se asocian habitualmente a resistencia, y su aparición se debe a características propias del virus como la elevada tasa variabilidad genética y capacidad replicativa. 
Tabla 2. Frecuencia y relevancia de mutaciones encontradas

\begin{tabular}{|llll|}
\hline Mutaciones & & Pacientes $\mathbf{n}=\mathbf{7 4}$ & Nivel de resistencia \\
Gen transcriptasa reversa & M41L & $1(1,4 \%)$ & Intermedia a AZT,D4T \\
& V118I & $4(5,2 \%)$ & Baja a AZT-D4T \\
& T215S & 1 & Intermedia/Baja a AZT-D4T \\
K103N & $1,4 \%)$ & Alta a NVP, EFV \\
V179D & $1(1,4 \%)$ & Intermedia a NVP, EFV \\
Gen proteasa & $2(2,8 \%)$ & Baja a todos los IP \\
& L10IN & $27(36,5 \%)$ & Baja a todos los IP \\
& I13V & $14(18,9 \%)$ & Baja a todos los IP \\
& G15V & $12(16,2 \%)$ & Baja a todos los IP \\
& L19I & $5(7,1 \%)$ & Baja a todos los IP \\
& K20R/M & $2(2,9 \%)$ & Baja a todos los IP \\
L33V & $8(11,4 \%)$ & Baja a todos los IP \\
M36I & $1(1,4 \%)$ & Baja a todos los IP \\
K43T & $17(23 \%)$ & Baja a todos los IP \\
D60E & $3(4,2 \%)$ & Baja a todos los IP \\
I62V & $5(7,1 \%)$ & Baja a todos los IP \\
L63P/T & $5(6,8 \%)$ & Baja a todos los IP \\
A71TN & $38(51,4 \%)$ & Baja a todos los IP \\
T74S & $9(12,8 \%)$ & Baja a todos los IP \\
V77I & $1(1,4 \%)$ & Baja a todos los IP \\
I93L & $26(35,1 \%)$ & Baja a todos los IP \\
\hline
\end{tabular}

*1 Paciente presentó mutaciones asociadas a resistencia elevada a una droga de la familia de los IP (amprenavir/fosamprenavir), debido a la presencia simultánea de las mutaciones L10I, L19I, I62V y L63P.

De éstas las más frecuentes fueron L63P/T (38 pacientes), L10I/V (27 pacientes) y V77I (26 pacientes) (Tabla 2).

\section{Discusión}

La resistencia primaria a las ARV ha sido bien documentada, sobre todo en países desarrollados, y su tendencia es variable dependiendo del área geográfica y de las características de los pacientes analizados ${ }^{7}$. La mayoría de los estudios realizados desde finales de la década de los noventa hasta los años 2003-2004 evidenciaron altos niveles de prevalencia de la resistencia primaria con una tendencia al $\mathrm{alza}^{12}$. Un estudio realizado entre los años 1995 y 2004 en Nueva York, en el que se enrolaron 316 pacientes naive a TARV y con infección reciente, muestra una prevalencia de resistencia primaria de 13,2\% entre los años 1995-1998 y una prevalencia de $24,1 \%$ para el período comprendido entre los años 2003-2004, evidenciando una clara tendencia al alza en los niveles de resistencia primaria, tal como se estaba reportando en otros estudios de la época ${ }^{7,15-18}$.
En Europa se describe una situación similar. En el Reino Unido se reportó un estudio multicéntrico realizado entre los años 1996 y 2003 , donde se analizaron muestras de 2.357 pacientes naive a TARV, que evidenció un aumento significativo en la prevalencia de resistencia primaria, alcanzando el nivel de prevalencia más bajo en 1998 (8\%), y el más elevado entre 2002-2003 $(19,2 \%)^{19}$. En otras partes de Europa se describen tendencias similares.

En los últimos años se ha evidenciado una tendencia a la estabilización de la resistencia primaria, incluso disminución de ésta en algunas clases de ARV. El tiempo de infección estimado desde el contagio hasta la realización del test de resistencia es crucial cuando analizamos los índices de resistencia primaria. Este punto no ha sido considerado en todos los estudios, dato que es valioso de analizar ya que con el tiempo, las cuasiespecies que presentan mutaciones asociadas a resistencia pueden no aparecer en el estudio genotípico ya que se elimina la presión selectiva ejercida por los ARV, y empieza a predominar la cepa wild type, que tiene mayor capacidad replicativa, y por lo tanto, estas cuasiespecies pueden no ser detectadas en el 
estudio genotípico al representar menos de 20\% de la población viral (poblaciones minoritarias) ${ }^{20}$. Las mutaciones asociadas a resistencia al grupo de los análogos de nucleósidos son las primeras en desaparecer una vez que se elimina la presión selectiva de ARV, principalmente la M184V (menos de 1 año) ya que contribuye negativamente en la capacidad replicativa del virus); en tanto las mutaciones asociadas a resistencia al grupo de los IP son las que más pueden persistir en el tiempo sin la presión selectiva de los ARV (más de 2 años) ${ }^{21-23}$. Estas mutaciones pueden dejar de ser detectadas en los test de resistencia, pero se mantienen en los reservorios, de modo que reaparecen al ejercer presión selectiva con $\mathrm{ARV}^{23}$.

En Sudamérica hay pocos estudios de resistencia primaria, y generalmente las casuísticas corresponden a grupos pequeños. Un estudio realizado en Argentina, realizó un análisis genotípico de 123 pacientes VIH naive a TARV, con un tiempo estimado de infección menor de 9 meses, provenientes de centros urbanos argentinos, reclutados durante los años 2004 a 2007. Los resultados arrojaron que $8,9 \%$ de los pacientes tenían mutaciones asociadas a resistencia a ARV: $4,9 \%$ para el grupo de los NNRTI, 2,4\% para el grupo de los NRTI, 0,8\% para el grupo de los IP (mutaciones mayores) y $0,8 \%$ para las 3 clases de drogas ARV. Otro estudio argentino evaluó la prevalencia de resistencia primaria en 284 pacientes reclutados entre los años 2003 a 2005, encontrándose 4,2\% de pacientes con mutaciones asociadas a resisten$\mathrm{cia}^{24}$. Un estudio brasileño evalúo la prevalencia de resistencia primaria en 444 pacientes vírgenes a TARV, provenientes de tres áreas de Brasil alcanzando ésta 7,2\%, siendo mayor en el grupo de los NNRTI (5,4\%), y luego en los NRTI (3,2\%). No se encontraron mutaciones asociadas con resistencia a los IP, y la resistencia a dos clases fue de a 1,4\%. Otras regiones de latinoamérica muestran niveles similares, con rangos que van entre los 3 y $9 \%{ }^{25}$.

El análisis de los resultados de nuestro estudio, muestra que la prevalencia total de mutaciones asociadas a resistencia primaria fue de $2,7 \%$. Estos resultados evidencian una prevalencia menor que países vecinos como Argentina y Brasil, aunque son resultados difíciles de comparar debido a la diferencia en el " $n$ " de los grupos de estudio.

$\mathrm{Si}$ analizamos por separado los grupos de ARV, $1,4 \%$ de los pacientes tiene mutaciones que le confieren elevada resistencia a los NNRTI, y hay 2,7\% que presenta mutaciones asociadas con resistencia intermedia a esta clase; $2,7 \%$ de los pacientes tiene un virus parcialmente resistente a los análogos de timidina, y 1,4\% tiene resistencia a un IP dada por la acumulación de ciertas mutaciones, a lo que se agrega que $17,6 \%$ de los pacientes tienen reducida la sensibilidad a algún IP. No se detectaron mutaciones asociadas con elevada resistencia a los IP, ni pacientes con mutaciones asociadas a resistencias de 2 o más clases de antirretrovirales. Casi la totalidad de los pacientes estudiados $(98,7 \%)$ presentó al menos una mutación, y más de $25 \%$ presentó 4 o más mutaciones. Los resultados evidenciados apoyan que, por el momento, no estaría recomendada la realización de un test de resistencia previo al inicio de la TARV en los pacientes naive, ya que la prevalencia total de resistencia primaria fue de $2,7 \%$, y está indicado en las guías internacionales cuando ésta es $\geq 5 \%$.

Se han publicado en Chile dos estudios previos a éste, analizando la prevalencia de resistencia primaria. El primero de ellos fue realizado entre 2001 y 2002, que analizó 60 pacientes controlados en el área norte de Santiago. Toda la población en estudio presentaba infección VIH crónica, y no habían recibido ARV ${ }^{26}$. Este estudio no utilizó el criterio de infección reciente para reclutar a los pacientes, que es factor fundamental cuando se evalúa resistencia primaria, por lo que la mayoría de ellos presentaba infección crónica de más de 18 meses de evolución desde el diagnóstico al momento de realizar el análisis genotípico (56,7\% etapa SIDA). Ninguna de las mutaciones encontradas se asoció a un elevado grado de resistencia a los NRTI, NNRTI o IP. En un segundo estudio, se analizaron 25 pacientes, reclutados entre 2004 y 2005, provenientes de la ciudad de Santiago, con diagnóstico reciente de infección VIH $(<18$ meses), y vírgenes a TARV ${ }^{27}$. La prevalencia de resistencia primaria fue de $12 \%$. Dentro de los antecedentes epidemiológicos de este estudio, es destacable que todos los pacientes en que se detectaron mutaciones, habían adquirido la infección por VIH en el extranjero debido a conductas de riesgo llevada a cabo en esos lugares (sexo desprotegido y/o uso de drogas endovenosas). Los países donde los pacientes habrían adquirido la infección, presentan prevalencias de resistencia primaria de un orden mayor al 10\%. Todos los pacientes estudiados, correspondían a personas con nivel educacional superior, pertenecientes al 
estrato socioeconómico alto, por lo que no es una muestra representativa de la población total de pacientes con infección VIH en Chile.

Consideramos que nuestro estudio es representativo de la realidad nacional, reconociendo como limitación el bajo número de pacientes enrolados. De acuerdo a nuestros resultados, no se recomienda realizar el test de resistencia en pacientes naive a TARV en Chile, debido a que la resistencia primaria tiene una prevalencia muy baja $(<5 \%)$.

A futuro la evolución de la resistencia primaria debería seguir siendo estudiada, incluyendo un mayor número de pacientes, para evaluar la tendencia nacional cada 3 años, y así poder contar con datos estadísticos locales al momento de hacer recomendaciones para el manejo de nuestros pacientes.

\section{Referencias}

1. Resumen mundial de la epidemia de SIDA, Diciembre de 2007. Disponible en www.unaids.org

2. Sepúlveda C. Terapia antirretroviral: Generalidades y descripción de Drogas. En: Sepúlveda C, Afani A, eds. SIDA. 3a edición, 2002.

3. Menendez-Arias L. Targeting HIV: antiretroviral therapy and development of drug resistance. TIPS 2002; 23: 3818.

4. Ministerio de Salud. Guía Clínica Síndrome de la Inmunodeficiencia Adquirida. VIH/SIDA. 1st Ed. Santiago: Minsal, 2005.

5. Petroni A, Deluchi G, Pryluka D. Update on Primary HIV-1 Resistance in Argentina: Emergence of Mutations Conferring High-Level Resistance to Nonnucleoside Reverse Transcriptase Inhibitors in Drug-Naive Patients. J Acquir Immune Defic Syndr 2006; 42: 50610.

6. Viani R. Prevalence of Primary HIV-1 Drug Resistance among Recently Infected Adolescents: A Multicenter Adolescent Medicine Trials Network for HIV/AIDS Interventions Study. J Infect Dis 2006; 194: 1505-9.

7. Chaix ML, Descamps D, Harzic M. Stable prevalence of genotypic drug resistance mutations but increase in non-B virus among patients with primary HIV-1 infection in France. AIDS 2003; 17: 2635-43.

8. Weinstock HS, Zaidi I, Heneine W. The epidemiology of antiretroviral drug resistance among drugnaive HIV-1 infected persons in 10 US cities. J Infect Dis 2004; 189: 2174-80.
9. Wensing AMJ, Van DE Vijver DA, Angarano G. Prevalence of drug-resistant HIV-1 variants in untreated individuals in Europe: implications for clinical management. J Infect Dis 2005; 192: 958-66.

10. Mark O. Primary HIV Drug Resistance and Efficacy of First-Line Antiretroviral Therapy Guided by Resistance Testing. J Acquir Immune Defic Syndr 2006; 41: 573 81.

11. Grubb J. Patterns of primary antiretroviral drug resistance in antiretroviral-naive HIV-1-infected individuals in a midwest university clinic. AIDS 2006; 20: 2115-25.

12. Hirsch M. Antiretroviral drug resistance testing in adults infected with HIV-1. 2003 recommendations of an IASUSA panel. Clin Infect Dis 2003; 37: 113-28.

13. Johnson VA, Brun-Vézinet F, Clotet B, et al. Update of the Drug Resistance Mutations in HIV-1: 2007. Top HIV Med 2007; 15: 119-25.

14. HIV Drug Resistence Mutations by Drugs Class (January 28, 2008). Disponible en http://hivdb.stanford.edu

15. Shet A, Berry L, Mohri H, Mehandru S, Chung C, Kim A. Tracking the prevalence of transmitted antiretroviral drugresistant HIV-1: a decade of experience. J Acquir Immune Defic Syndr 2006; 41: 439-46.

16. Oette M, Kaiser R, Daumer M, Petch R, Fatkenheuer G, Carls H. Primary drug resistance and efficacy of firstline antiretroviral therapy guided by resistance testing. J Acquir Immune Defic Syndr 2006; 41: 573-81.

17. Grant RM, Hecht FM, Warmerdam M, Liu L, Liegler T, Petropoulos CJ. Time trends in primary HIV-1 drug resistance among recently infected persons. JAMA 2002; 288: $181-8$.

18. Little SJ, Holte S, Routy JP. Antiretroviral-drug resistance among patients recently infected with HIV. N Engl J Med 2002; 347: 385-94.

19. UK Group on Transmitted HIV Drug Resistance. Time trends in primary resistance to HIV drugs in the United Kingdom: multicentre observational study. BMJ 2005; 331: 1368.

20. Hirsch M, Gunthard H, Schapiro J. Antiretroviral Drug Resistance Testing in Adult HIV-1 Infection: 2008 Recommendations of an International AIDS Society-USA Panel. Clin Infect Dis 2008; 47: 266-85.

21. Bezemer D, De Ronde A, Prins M. Evolution of transmitted HIV-1 with drug-resistance mutations in the absence of therapy: effects on CD4+ T cell count and HIV-1 RNA load. Antivir Ther 2006; 11: 173-8.

22. Brenner B, Routy JP, Quan Y. Persistence of multidrug resistant HIV-1 in primary infection leading to superinfection. AIDS 2004; 18: 1653-60.

23. Ghosn J, Pellegrini I, Goujard C. HIV-1 resistant strains 
acquired at the time of primary infection massively fuel the cellular reservoir and persist for lengthy periods of time. AIDS 2006; 20: 159-70.

24. Dilernia D, Lourtau L, Gómez A. Drug-resistance surveillance among newly HIV-1 diagnosed individuals in Buenos Aires, Argentina. AIDS 2007; 21: 1355-60.

25. umans A, Soares M, Pieniazek D, Kalish M. Prevalence of Protease and Reverse Transcriptase Drug Resistance Mutations over Time in Drug-Naïve Human Immuno- deficiency Virus Type 1-Positive Individuals in Rio de Janeiro, Brazil. Antimicrob. Agents Chemother 2002; p. 3075-9.

26. Afani A, Ayala M, Meyer A, Acevedo W, Cabrera R. Resistencia primaria a terapia antirretroviral en pacientes con infección por VIH/SIDA en Chile. Rev Med Chile 2005; 133: 295-301.

27. Acevedo W, Gallardo AM. Detección de resistencia primaria en chilenos infectados por VIH. Rev Med Chile 2007; 135: 1406-13. 